\title{
PLANE-WAVE ANALYSIS OF SOI DATA
}

\author{
R. S. BOGART AND L. A. DISCHER DE SÁ
}

Stanford University

I. GONZÁLEZ HERNÁNDEZ AND J. PATRÓN RECIO

Instituto de Astrofísica de Canarias

D. A. HABER AND J. TOOMRE

$J I L A$

F. HILL

National Solar Observatory

E. J. RHODES, JR. AND Y. XUE

University of Southern California

AND

THE SOI RING DIAGRAMS TEAM

\begin{abstract}
.
The unprecedented combination of spatial resolution and stability achieved by the Solar Oscillations Investigation/Michelson Doppler Imager on SOHO has opened up new opportunities for the analysis of solar surface oscillations of high spatial frequencies. In this regime the oscillations are essentially plane waves, amenable to the techniques of ring-diagram analysis of their three-dimensional power spectra. This approach holds the promise of measuring fluid motions and possibly magnetic fields in spatially-resolved structures within the uppermost levels of the convective envelope, a region unresolved by the global modes. Atmospheric $g$-modes trapped above the photosphere may also be detectable. We review the first results of planewave analysis of various types of SOI data and comparisons with the analyses of comparable ground-based datasets.
\end{abstract}




\section{Introduction}

A fundamental goal of local-area helioseismology is to subject regions smaller than the entire visible part of the Sun to analysis for any peculiar velocity fields, radial structure variations, and strong magnetic fields that may exist at certain times and differentiate them from other such regions. The most straight-forward technique is to apply what was historically the earliest helioseismic method, plane-wave analysis, to suitably remapped and tracked observations. The approach has been described and elaborated elsewhere: see for example Hill(1988) and Patrón(1994). The principle justification for plane-wave analysis of eigenmodes trapped in spherically-symmetric cavities is that the regions considered are so small that the curvature of the cavity is negligible and the waves observed are of such short lifetimes that a spherical closure condition scarcely applies. The numerical simplicity of a three-dimensional Fourier transform strongly recommends itself over spherical harmonic decomposition when the effective spherical harmonic degree of the eigenmodes may be well over 1000 and the region sampled less than $1 \%$ of the surface of the sphere.

It has been demonstrated that three-dimensional plane-wave analysis can be applied to observations of solar oscillations obtained with highresolution instruments on the ground (Hill, 1988; Patrón, 1994; González et $a l ., 1995)$. Inversion of these results for the sub-photospheric velocity fields yields at least plausible pictures of flows in the upper convective envelope, a region inadequately resolved by global-mode helioseismology. Long-period ground-based observations are severely affected by atmospheric seeing on the scale of the wavelengths of the modes of interest, however. In order to verify that ground-based high-resolution oscillation measurements are not systematically biased or distorted by the observing conditions, it is essential to observe the same regions at the same time from several instruments and ideally from space. In the absence of significant pointing jitter and drift, space-based observations will also allow us to begin to construct synoptic views of the Sun, by obtaining data for long periods under virtually unchanging conditions, and to extend the observations to even higher spatial frequencies. Since the commencement of its scientific observing programme in April 1996 the Solar Oscillations Investigation/Michelson Doppler Imager (SOI/MDI) on Soho has been providing a wealth of data ideally suited to these tasks. The development and application of planewave analysis techniques has already proceeded from various instrument commissioning observations (Bogart et al., 1995b). Here we summarize the results of the first analyses of calibrated data sequences from SOI/MDI and the comparisons with concurrent ground data. 


\section{Analysis Techniques}

The principle diagnostic of plane-wave analysis thus far is the horizontal flow field, manifested by dipole terms in the expansions of the eigenfrequencies $\omega_{n}(\theta)$ for a given $k$. (For a uniform transverse motion of the observer, advection of the wave fronts leads to a shift in the measured mode frequencies of $\left.\Delta \omega_{n}(k)=v \cdot k\right)$ To remove the $2 \mathrm{~km} / \mathrm{s}$ signature of solar rotation and to follow identified photospheric features, we select small regions centered at selected Carrington coordinates at a given time. The regions are tracked at the rate of photospheric differential rotation appropriate for the latitudes of their central points (Snodgrass, 1984).

The selected regions are mapped from the plate coordinates via Postel's projection, a standard cartographic projection in which path lengths along lines through the center of the map are proportional to lengths along the corresponding geodesics. Since the regions selected for analysis are small, typically about $15^{\circ}$ in diameter, the choice of projection is not critical. The map scale is selected to preserve the instrumental resolution at the center of the disc: for MDI full-disc images this is 1.978 arc-sec per pixel, corresponding to a map scale of $0^{\circ} .12$ heliographic per pixel. The resulting maps of the line-of-sight Doppler velocities (or other observed parameters) are apodized in a circular region using an exponential taper with a characteristic thickness of 3.5 pixels to equalize the spatial frequency resolution in all directions.

In order to improve the convergence of some of the fitting procedures, the tracked and mapped data are generally high-pass filtered in the time domain using a gaussian window of full-width-half-maximum 21 minutes $(0.8 \mathrm{mHz})$, after which the three-dimensional power spectrum is computed. A sample power spectrum of a 2-day interval of SOI/MDI Doppler data is shown in Figure 1. The eigenmodes form the characteristic set of nested trumpet surfaces, the outermost being the lowest order modes.

There are two basic approaches to analyzing the power spectrum. In the standard technique, described by Hill(1988) and substantially improved by Haber et al.(1995) and Patrón et al.(1996), the variations are analyzed along cuts in temporal frequency, where the eigenfrequencies appear as sets of nearly circular concentric rings (hence the name "ring-diagram analysis"). Directional dependencies of the sound speed and/or frequency shift show up as asymmetries in the rings; these asymmetries may be parametrized and measured for particular eigenmodes as functions of frequency. An alternative approach, suggested by Bogart et al.(1995a), is to cut the power spectrum along sets of concentric cylinders in the two-dimensional spatial frequency space. Along such cylinders the frequency shifts of particular eigenmodes depend on the azimuthal angle; they can be Fourier-decomposed 


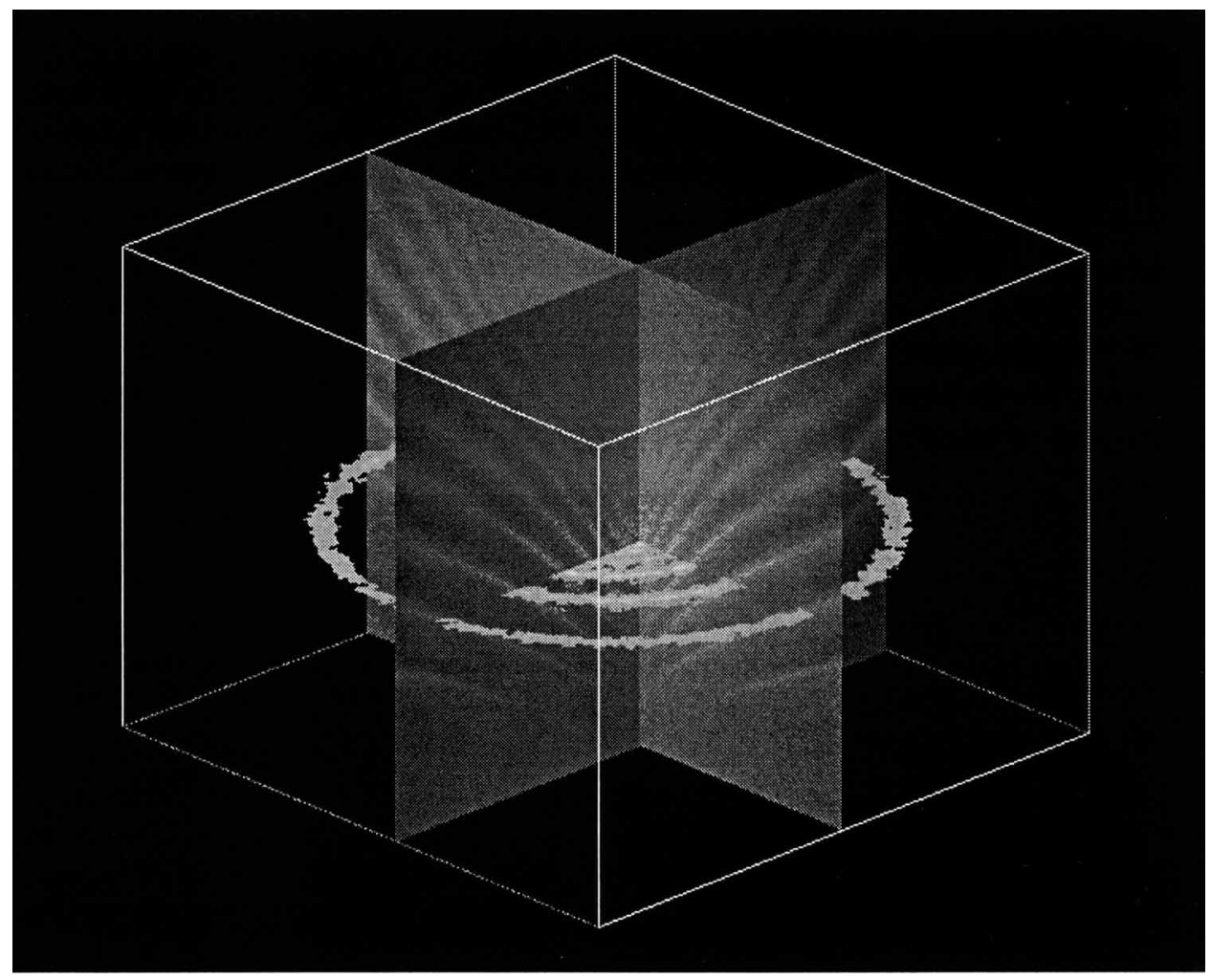

Figure 1. Three-dimensional Doppler power spectrum from SOI/MDI. The region observed is concentric and coterminous with region $\mathrm{F}$, but of twice the diameter (region $\mathrm{F}^{\prime}$ ). Power is shown projected along the horizontal axes, corresponding to orthogonal components of the horizontal wavenumber, and on a single cut in the vertical (temporal frequency) axis.

to isolate the first-degree advection terms from the second-degree sound speed variation terms. The two approaches are obviously complementary, the first having its greatest resolving power for lower spatial frequencies (higher-order modes) and the second for higher spatial frequencies (lowerorder modes). Considerable effort has been made to refine and compare these approaches with the aim of systematizing plane-wave analysis in future. The results presented at this meeting rely predominantly on the standard ring-fitting approach, but have been corroborated in a few cases with the Fourier approach.

To infer the depth dependence of the horizontal velocity field from the first-order terms in the frequency shifts, we perform a least-squares inversion with second derivative smoothing, as described by Patrón(1994). This inversion procedure allows us to tune the trade-off parameter between res- 
olution and error magnification, an essential step because of the current uncertainties in our error estimation procedures.

\section{Data Analyzed}

The various regions analyzed are summarized in Table 1 . All are quiet sun regions except for regions $\mathrm{B}$ and $\mathrm{C}$, which cover a predominantly unipolar area of a decaying active region and a sunspot, respectively. The latitude and longitude of disc center $\left(b_{0}, l_{0}\right)$ are nominal, referred to the center of the tracking interval. In all cases the regions analyzed were circles on the photosphere of diameter $15^{\circ} .36$ heliographic or $186.6 \mathrm{Mm}$.

TABLE 1. Regions Selected for Analysis

\begin{tabular}{|c|c|c|c|c|c|c|}
\hline & Start Time & End Time & $b_{C}$ & $l_{C}$ & $b_{0}$ & $l_{0}$ \\
\hline $\mathrm{A}$ & 96.06 .06 14:17:30 & 96.06 .07 07:20:30 & 3.04 & 291.0 & 0.04 & 287.8 \\
\hline B & 96.06 .06 14:17:30 & 96.06 .07 07:20:30 & -4.96 & 268.2 & 0.04 & 287.8 \\
\hline $\mathrm{C}$ & 96.06 .06 14:17:30 & 96.06 .07 07:20:30 & 5.04 & 305.8 & 0.04 & 287.8 \\
\hline $\mathrm{D}$ & 96.06 .02 12:59:30 & 96.06 .03 06:02:30 & -0.45 & 341.4 & -0.45 & 341.4 \\
\hline $\mathrm{E}$ & $96.06 .0212: 59: 30$ & 96.06 .03 06:02:30 & -0.45 & 26.4 & -0.45 & 341.4 \\
\hline $\mathrm{F}$ & $96.06 .0106: 09: 30$ & 96.06 .03 06:44:30 & -0.53 & 349.7 & -0.53 & 349.7 \\
\hline
\end{tabular}

Doppler data were extracted for all regions. In addition, line-depth (equivalent-width) measurements exist for the same intervals, but these have not yet been analyzed. Concurrent ground-based observations have been obtained during the selected intervals by various combinations of the Taiwan Oscillations Network (TON), the High- $l$ Helioseismometer at Kitt Peak, and the High-Degree Helioseismology Network (Mt. Wilson). So far, the only detailed comparison made has been with a 512-minute subsequence of region F (González Hernández et al., 1996).

\section{Results}

The inversions for the first-order terms in the expansion of the local frequency shifts, sensitive to the horizontal velocity profile, are in general agreement with those seen before in ground data, at least for quiet sun regions. The detailed comparison with the results from a set of concurrent ground-based obsevations, reported elsewhere in these proceedings (González Hernández et al., 1996), is quite close. The results for regions of moderate magnetic activity, also reported elsewhere (Haber et al., 1996) are less clear. There is apparent spatial asymmetry in the observed power 

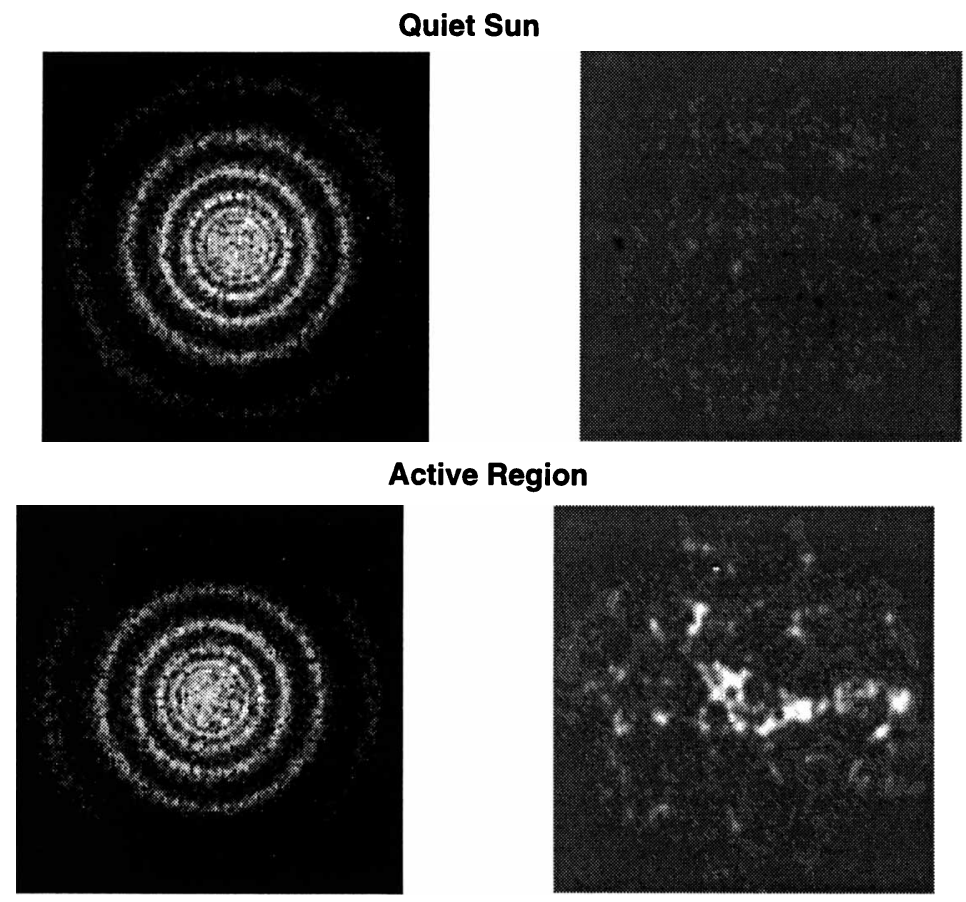

\section{Sunspots}
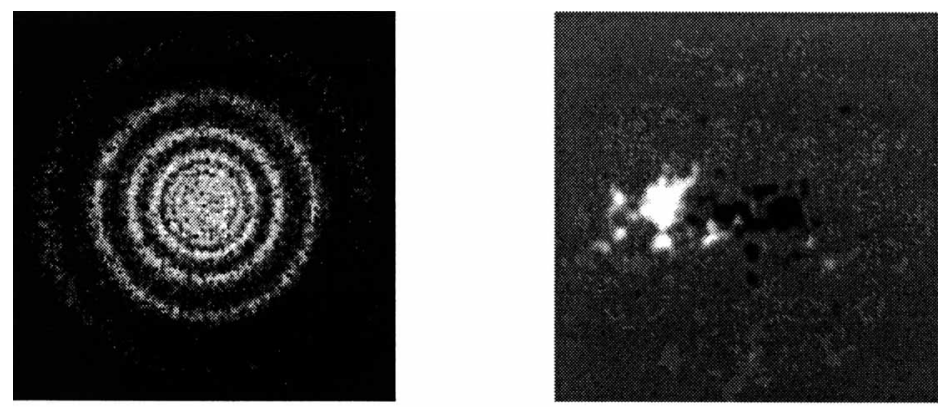

Figure 2. Ring diagrams and the corresponding magnetograms for regions A-C.

distribution in the ring diagrams for the magnetically active regions in Figure 2, but similar effects are seen when quiet-sun observations at different distances from disc center are compared (regions D \& E). Some of this effect is undoubtedly due to foreshortening, and the extent to which it affects measurement of the ring-shift parameters is uncertain.

Attempts to measure the second-order terms, sensitive to directional 


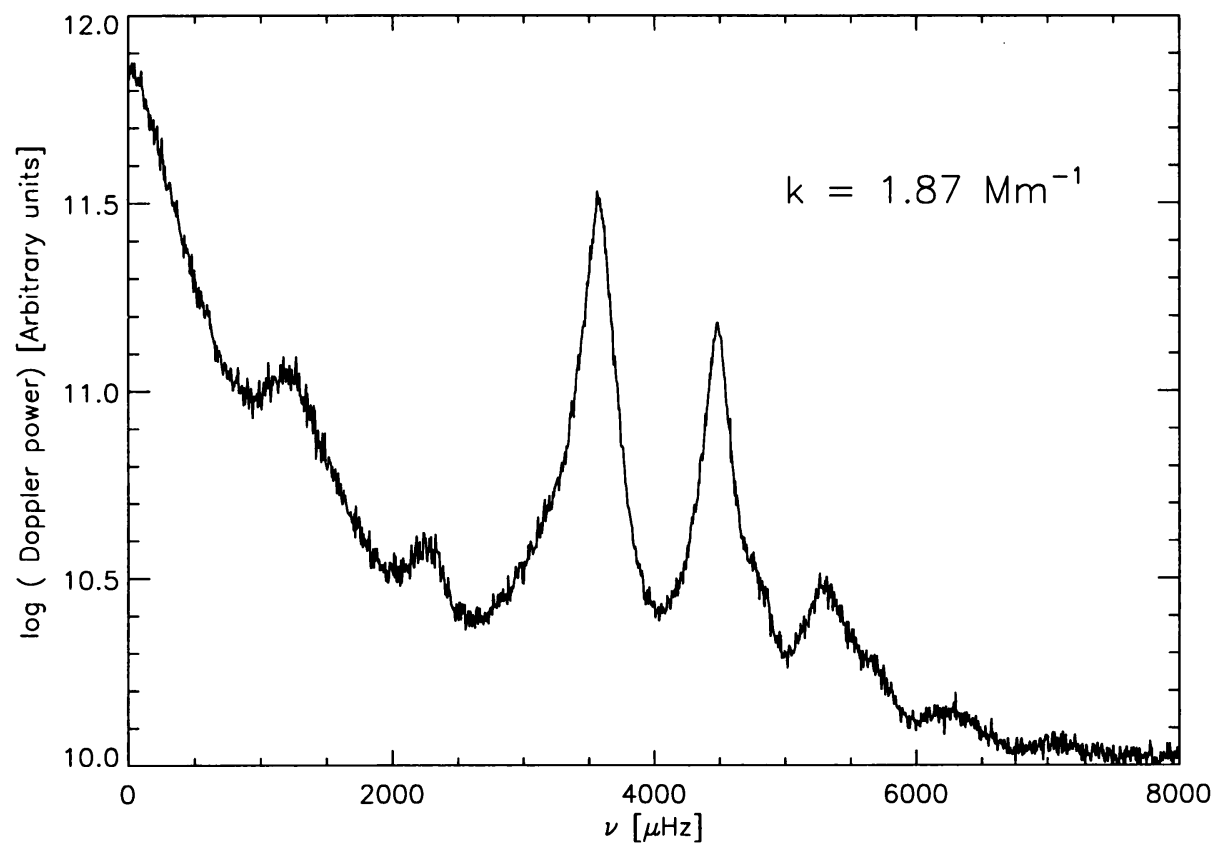

Figure 3. Temporal frequency spectrum of the azimuthally averaged Doppler power for region $\mathrm{F}^{\prime}$ for a value of $k$ equal to $1.87 \mathrm{Mm}^{-1}$. No temporal filtering was performed.

sound speed variations and magnetic field effects, are also reported elsewhere here (Haber et al., 1996). There is no conclusive detection of such effects in the samples studied.

At very high spatial frequencies we observe spectral features at temporal frequencies below those of the acoustic waves as the period of the $f$-mode decreases to below the 5-minute band. In the azimuthally-averaged power spectrum shown in Figure 3, corresponding to an effective degree of $l \approx 1300$ for global modes, two such features are clearly visible. There are hints in the preliminary analysis that the higher-frequency feature, here appearing at about $2.3 \mathrm{mHz}$, is dispersive; it might be a signature of atmospheric $g$-modes. The other feature, at a frequency of about $1.2 \mathrm{mHz}(P \approx 14$ min) nearly independent of $k$, seems to vary in strength significantly with magnetic activity, being enhanced by a factor of roughly 30 in regions B \& $\mathrm{C}$ over region A. Formal identification of these features is premature, but there is at least a strong hint that we may be beginning to systematically sound not just the sun's interior, but its atmosphere as well. 


\section{Acknowledgments}

SOHO is a project of international cooperation between ESA and NASA. This research is supported by the SOI-MDI NASA contract NAG5-3077 at Stanford University.

\section{References}

Bogart, R. S., Sá, L. A. D., Duvall, T. L., Haber, D. A., Toomre, J. and Hill, F. (1995) Plane-Wave Analysis of Solar Acoustic-Gravity Waves: A Slightly New Approach. Proceedings of the Fourth Soho Workshop, on Helioseismology, Pacific Grove, California, USA, 2-6 April 1995 (ESA SP-376), Vol. 2, pp. 147-150.

Bogart, R. S., Sá, L. A. D., Haber, D. A., and Hill, F. (1995) Preliminary Results From Plane-Wave Analysis of Helioseismic Data. Proceedings of the Fourth Soho Workshop, on Helioseismology, Pacific Grove, California, USA, 2-6 April 1995 (ESA SP-376), Vol. 2, pp. 151-152.

González, I, Patrón, J, and the TON TEAM (1995) Ring Diagram Analysis With TON: Preliminary Results. Proceedings of the Fourth Soho Workshop, on Helioseismology, Pacific Grove, California, USA, 2-6 April 1995 (ESA SP-376), Vol. 2, pp. 137-139.

González Hernández, I, Patrón, J, Bogart, R., Sá, L., and the TON and SOI Teams (1996) Comparison of SOI and TON Data With Ring Diagram Techniques Sounding Solar and Stellar Interiors: IAU Symposium 181 Nice, France, 30 Sep. - 3 Oct 1995. (in press).

Haber, D. A., Toomre, J., Hill, F., and Gough, D. O. (1995) Local-Area Analysis of HighDegree Solar Oscillations: New Ring Fitting Procedures. Proceedings of the Fourth Soho Workshop, on Helioseismology, Pacific Grove, California, USA, 2-6 April 1995 (ESA SP-376), Vol. 2, pp. 141-146.

Haber, D., Zweibel, E., Toomre, J., Bogart, R. S., Sá, L., Burnette, A., Xue, Y., Rhodes, E., and Hill, F. (1996) Possible magnetic field effects seen with local-area analysis of MDI and Mount Wilson data. Sounding Solar and Stellar Interiors: IAU Symposium 181 Nice, France, 30 Sep. - 3 Oct 1995. (in press).

Hill, F. (1988) Rings and Trumpets- Three Dimensional Power Spectra of Solar Oscillations. Ap.J., 333, pp. 996-1013.

Patrón Recio, J. (1994) Tridimensional Distribution of Horizontal Velocity Flows Under the Solar Surface. Doctoral Dissertation, Departamento de Astrofisica, Universidad de La Laguna.

Patrón, J, González Hernandez, I, and the TON TEAM (1995) Comparisons on fitting ring diagrams. Sounding Solar and Stellar Interiors: IAU Symposium 181 Nice, France, 30 Sep. - 3 Oct 1995. (in press).

Snodgrass, H.B. (1984) Separation of Large-Scale Photospheric Doppler Patterns. Solar Phys., 94, pp. 13-31. 